\title{
Aisthesis
}

Firenze University Press

www.fupress.com/aisthesis

OPEN ACCESS

Citation: G. Ferrario (2021) Vaga lume. Estetica della transizione. Aisthesis 14(1): 165-172. doi: 10.36253/Aisthesis- 11345

Copyright: ( $92021 \mathrm{G}$. Ferrario. This is an open access, peer-reviewed article published by Firenze University Press (http://www.fupress.com/aisthesis) and distributed under the terms of the Creative Commons Attribution License, which permits unrestricted use, distribution, and reproduction in any medium, provided the original author and source are credited.

Data Availability Statement: All relevant data are within the paper and its Supporting Information files.

Competing Interests: The authors have declared that no competing interests exist.

\section{Firefly. Aesthetics of transition}

\author{
Vaga lume. Estetica della transizione ${ }^{1}$
}

\section{Giovanni Ferrario}

Università Cattolica di Milano

giovanni.ferrario@unicatt.it

\begin{abstract}
This essay starts with the assumption that philosophical practice is defined as an exercise of linguistic clarification. Whether in oral or written form, philosophy stems from the need to explicate and objectify the logós, formulating concepts. In the particular case of artistic practice, on the other hand, the signifier is more visible than the signified and the impermanence of objectivity is more clearly shown. In art, language is thus open to multiplicity and inexpressibility and the word is no longer logically or syntactically objectified. The truth which develops within art is not universal but the embodiment of an engaging and embryonic form. Art is the place where truth is in motion, a form of resistance, an all-embracing, unifying experience intimately close to world- changing practices.
\end{abstract}

Keywords: Transit, Truth, Logós, Clarification, Objectivity.

O dolce lucciola, bubola o borboleta fosforica, che t'industri ad accecarmi con pirotecnie di fantascienza da quattro soldi nelle penombre che più non valgono un soldo per non dire quando ti atteggi a fiamma ossidrica tu drago che basta il fiato d'un bimbo a estinguere (Zanzotto [2011]: 629)

\section{LE FILOSOFIE EDIFICANTI. PRECISAZIONE E SIGNIFICATO.}

Uno dei caratteri che intimamente muove la pratica filosofica è il tentativo di precisare concetti. Precisare è scavare nel linguaggio,

${ }^{1}$ Il saggio nasce dal gentile invito di Roberta Corvi a tenere una lezione con Roberto Diodato sulla questione dell'oggettività nell'arte, nell'ambito delle lezioni di Teoria della conoscenza in età contemporanea presso l'Università Cattolica del S. Cuore di Milano, il 9 aprile 2019. Da tale sollecitazione germoglia questa riflessione contenuta nel presente saggio che vuole essere un timido ringraziamento per gli stimoli espressi in quelloccasione. 
descrivere le tracce antropiche, aprirsi all'archeologia del significato, astrarre dal mondo i concetti che ne rappresentano il senso, definire il luogo della parola. A partire dagli antichi sistemi fonetici orientali (hiragana e katakana) ${ }^{2}$, passando dal logocentrismo occidentale di matrice socratica o dal verbo vivo evangelico (kèrygma), il logós che si muta in scrittura delinea un orizzonte metafisico in cui la parola fonetica in presenza si trasforma in traccia dell'assenza. Si tratta di un'assenza che pesa, come ricorda Derrida, perché mette potenzialmente in crisi il sistema metafisico tradizionale resosi visibile nella traccia scritta come alterità irrappresentabile. "Se il momento nonfonetico minaccia la storia e la vita dello spirito come presenza a sé nel soffio, è perché minaccia la sostanzialità, altro nome metafisico della presenza, dell'ousia». (Derrida [1967]: 30). Che sia voce (phoné) o iscrizione (gràmma) nella filosofia vi è comunque il desiderio di precisare il logós, creando concetti e concatenazione di concetti, scoprendo nuove e inattese curvature lungo gli assi cartesiani della domanda.

Il concetto è sì una soluzione, ma il problema a cui risponde risiede nelle sue condizioni di consistenza intensionale e non, come nella scienza, nelle condizioni di referenza delle proposizioni estensionali. Se il concetto è una soluzione, le condizioni del problema filosofico devono stare sul piano di immanenza che esso presuppone (a quale movimento infinito rinvia nell'immagine del pensiero?) e le incognite del problema nei personaggi concettuali che esso mobilita (quale personaggio precisamente?) (Deleuze, Guattari [1991]: 72).

In tali incognite risiede la complessità dei personaggi concettuali creati e, benché la filosofia

\footnotetext{
${ }^{2}$ Per approfondimenti sullo sviluppo dei due sistemi sillabici indicati, si rimanda in modo specifico alla storia del pensiero tradizionale sino-giapponese (Ghilardi [2018]: 25-72), nonché all'analisi della costruzione ideografica nel pensiero giapponese classico (Raveri [2014]: 13-17). In modo particolare, l'impiego del sillabario katakana per trascrivere foneticamente le parole straniere ha portato la lingua giapponese a essere molto permeabile all'influsso di altri linguaggi, soprattutto nell'ultimo secolo.
}

non contempli, non rifletta né comunichi, essa non può fare a meno di precisare e chiarificare la contemplazione, la riflessione e la comunicazione. Anche per Wittgenstein lo scopo della filosofia è il rischiaramento logico dei pensieri. Non possiamo pensare a questa pratica come a una dottrina ma come un'attività. «Un'opera filosofica consta essenzialmente di chiarificazioni. Il risultato della filosofia sono "non proposizioni filosofiche", ma il chiarificarsi di proposizioni. La filosofia deve chiarire e delimitare nettamente i pensieri che altrimenti sarebbero torbidi e indistinti» (Wittgenstein [1961]: 50).

Buona parte della tradizione filosofica occidentale ci mostra il filosofo come un soggetto che si aggira nel labirinto dei significati, una figura che abita la crisi permanente del logós e che da tale pratica risulta vincolato. Wittgenstein ricordava bene che non possiamo uscire dalla gabbia del linguaggio poiché «i limiti del mio linguaggio significano i limiti del mio mondo» (Wittgenstein [1961]: 88). Da questo punto di vista la filosofia, dopo aver assolto alla sua funzione di delimitare l'ambito del dicibile e dell'indicibile, deve essere abbandonata come un attrezzo che ha svolto il suo compito.

Ciò non significa che tutto quello che non si può esprimere con la precisione della scienza o della logica filosofica sia da rifiutare e ignorare. Wittgenstein è anzi convinto che quello che trascende l'orizzonte definito dalla scienza e dalle precisazioni linguistiche, in un rapporto di verificabilità con i fatti del mondo, comporti questioni fondamentali per l'uomo. Infine, la precisazione linguistica è inadeguata per dire l'inesprimibile e l'ineffabile e, tuttavia, questi oggetti del pensiero restano come sospesi nella mente quali concetti che infinitamente chiedono precisazione.

In Nietzsche la questione della precisazione si fa critica, poiché il genio costruttivo dell'uomo, e in particolare del filosofo, è certamente capace di determinare notevoli e complicatissimi edifici teoretici e tuttavia, in questa fitta ragnatela di speculazioni, egli non deve scordarsi che tale ricerca è una sorta di adeguamento del mondo ai concetti che lui stesso crea, in una costante metamorfosi del mondo nell'uomo. 
La grande costruzione dei concetti mostra la rigida regolarità di un colombario romano e manifesta nella logica quel rigore e quella freddezza che sono propri della matematica. Chi è ispirato da questa freddez$z a$ difficilmente crederà che anche il concetto - osseo come un dado, spostabile e munito di otto vertici come questo - sussista unicamente come il residuo di una metafora, perché l'illusione del trasferimento artistico di uno stimolo nervoso in immagini, se non è la madre, (è) tuttavia la nonna di ogni concetto (Nietzsche [1873]: 22).

Vi sono filosofi che, avendo compreso tale rischio e tale potenzialità linguistica, hanno sospinto la parola verso un orizzonte evocativo generando e rigenerando concetti in modo quasi poetico. Platone, la cui opera è attraversata dalla consapevolezza della necessità di tale scardinamento linguistico, è tra gli esempi più potenti prodotti dall'Occidente. La filosofia non aiuta a rivelare ma a ricordare (anàmnesi). Nella pratica filosofica l'idea si libera come parola dal nucleo più intimo della realtà e rivendica il suo statuto di nominazione attingendo a una memoria originaria e fondativa.

Platone chiarisce in due opere quanto possano essere potenti l'idea e il linguaggio nel loro farsi precisazione, nel loro sviluppo di verità all'interno del dialogo filosofico. Nel Cratilo, in cui il filosofo ritiene il linguaggio una produzione umana diretta alla conoscenza delle idee e, in particolare, nel Fedro ove lo si mostra come una mirabile invenzione: «Ecco una conoscenza, mio re, che renderà gli Egiziani più sapienti e più abili a ricordare; grazie ad essa è stato trovato il farmaco per la memoria e per la sapienza» (Platone [2015]: 181). Tuttavia, è proprio la scrittura come pharmakon a essere messa in discussione poiché, come tutte le medicine, possiede terribili controindicazioni: è cura e veleno e ripete senza sapere.

Lo ricorda bene Derrida in alcune parti de La Dissémination (Derrida [1972]: 103-197). Nella rivisitazione del mito di Teuth e Thamus il filosofo francese fa parlare personaggi concettuali, frammenta le immagini di verità, semina tracce. Si tratta di una polisemia irregimentabile che vede nel logós il volto nascosto della verità e del suo mistero. "Il logós è dunque la risorsa, bisogna volgersi verso di esso, e non solo quando la sorgente solare è presente e rischia di bruciare gli occhi se li fissiamo in essa; bisogna rivolgersi verso il logós anche quando il sole sembra assentarsi nella sua eclisse. Morto, spento o nascosto, quell'astro è più pericoloso che mai» (Derrida [1972]: 120). Dunque per Platone, da questa particolare prospettiva, il logós diviene "soggetto" e "argomento"3, e le idee sono delle stelle attorno alle quali orbitano infiniti astri. In questo cosmo ideale ogni stella è legata (lèghein) al tutto.

Nel suo precisare, che si esprime nella trasformazione delle "cose" del mondo sensibile in paradigmi ideali, il logós si trasfigura nel mythos, arginando il rischio della delimitazione nominale, della chiusura del linguaggio in un unico significato monolitico. Nonostante Platone proponga una sorta di oggettivizzazione della realtà sensibile nelle idee riesce, attraverso il dialogo poetico, a far fiorire concetti complessi evocando immagini eterne quali oggetti puri della logica. Come la natura proteiforme delle immagini suggerisce, esse restano aperte, operative, potenziali. Tali figure concettuali non possono fare a meno dell'immagine da cui tali concetti nascono. Così, non possiamo più scindere i concetti originari di mimesi, metessi, giustizia, parusia, bene, dal Mito della caverna. Il supporto dell'immagine poetica è il veicolo della necessità del concetto.

In questo caso, la potenzialità di un siffatto linguaggio filosofico tenta di evadere dalla mera precisazione per divenire attenzione, un nuovo modo di avvicinarsi spontaneamente all'anima. In Platone il significato è in equilibrio col significante, lo abita e lo dischiude e il concetto sublima il suo precisarsi linguistico nell'oggetto figurale. Questa particolare prassi filosofica edificante, come direbbe Rorty, la ritroviamo anche in Eraclito di Efeso. Ogni radice ama nascondersi e nel crinale che unisce il dentro e il fuori di ogni

\footnotetext{
${ }^{3}$ In questo caso Derrida si conforma alla traduzione che Léon Robin fa del termine "idea". Si rimanda per maggior chiarezza ad alcune pagine del testo Platon (Robin [1968]: 77-82).
} 
pratica teoretica conficcata nella realtà si giunge al perpetuo mutare delle cose e con esse della loro verità. Filosofi come Bergson, Deleuze, Kierkegaard, Nietzsche, Derrida, Foucault, Wittgenstein, Benjamin attraverso racconti, aforismi e disseminazioni stanno sul bordo del linguaggio filosofico e accarezzano quello poetico. È questo luogo di confine che si affaccia sul buio del mondo che presenta la metamorfosi di cui l'arte ci parla, dischiudendo la piccola luce che sempre vacilla.

Lo stile filosofico, come lo chiamava Deleuze, è etica che vive nell'estetica. Pensare questa congiuntura in una trasposizione allusiva è un'apertura all'imprevisto e una via di fuga da ogni registrazione definitiva. Si tratta dunque della necessità di stare sulla linea del pensiero e di saperla spezzare, ritrovandosi in mare aperto, per inventare di nuovo l'impensabile, per raccogliere i bagliori fuggevoli di una vita.

\section{POTENZIALITÀ ACQUATICA. SIGNIFICANTE E TRANSITO.}

La pratica artistica manifesta con maggior chiarezza il transitare dell'oggettività (intesa come eidos, forma, idea) perché apre il linguaggio al molteplice e all'indicibile (Das Mystische) e non ferma la parola, non vuole oggettivarla o precisarla in termini logici e sintattici. La parola diviene figura, si trasforma in un'immagine intima, potenziale e solitaria che si apre alla relazione.

Nell'esperienza artistica incontriamo la potenza sospesa del gesto, della parola e della sua contemplazione. Viviamo un'esperienza originaria del mondo in cui soggetto e oggetto, essere e nonessere sono riassorbiti in un'unità da cui lo stesso pensiero nasce. Si tratta di un luogo (basho $)^{4}$

\footnotetext{
${ }^{4}$ Come indica puntualmente M. Ghilardi, questo orizzonte illimitato di accoglienza ricorda, in un rapporto di analogia, il concetto occidentale di "place", "lieu", "topos", "Ort", "lugar" a partire dalla tradizione platonica relativa al concetto di chóra. Per approfondimenti si vedano alcuni passaggi del testo di Ghilardi sull'unità di buono, vero e bello nell'estetica di Nishida Kitarō (Ghilardi [2009]: 219).
}

inoggettivabile, un fondo senza sfondo pronto a raccogliere ogni forma. Il filosofo giapponese Nishida Kitarō ricorda acutamente che «affinché la coscienza e l'oggetto stiano in relazione, deve esserci qualcosa che contiene entrambi in sé. Deve esserci qualcosa come un luogo in cui entrambi stanno in relazione. La vera forma delle forme deve essere il luogo delle forme» (Nishida [2012]: 31-32).

Tale territorio è la casa d'origine, «ove tutte le metafore e tutti i tropi vogliono essere condotti ad absurdum» (Celan [1983]: 17) e dove il significante risalta sul significato. É, secondo l'espressione della prima lettera ai Corinzi $(15,28)$ la "restaurazione di tutte le cose" (apokatàstasis pànton), ossia il tentativo di trovare nella nostra esperienza del mondo qualcosa d'immateriale e terrestre insieme, qualcosa di circolare, un luogo in cui essere e nulla si costituiscono infinitamente al suo interno. Edgar Morin ricorda un discorso dello scrittore francese Patrick Chamoiseau, tenuto il 2 giugno 2016 in occasione dell'esposizione dell'opera di Ernest Breleur alla Fondation Clémant, in cui si parla del gesto artistico come di un qualcosa di radicale e fondamentale; un qualcosa che è "una porta che si apre, che mai più si richiuderà, e che (ci) trasmetterà senza fine le energie dell'impossibile-da-concepire» (Morin [2016]: 112). L'arte è dunque un evento costruito da relazioni, un luogo di sintesi radicale che potenzialmente può essere ovunque e che porta con sé, attraverso una forma in transito, la radice delle forme.

Senza fenomeni, senza opere non ci sarebbe luogo, ma tali fenomeni esistono in virtù del luogo e del loro accadere. L'opera d'arte è come il fiore descritto da Angelus Silesius nel Pellegrino cherubico: «La rosa è senza perché (ohne Warum), fiorisce perché fiorisce; non pensa a sé, non si chiede se la si veda oppure no» (Silesius [1905]: 157). È quel mistero che rende muti, è l'immagine di un tremito che manifesta silenziosamente ogni verità, è quella figura inquieta che presenta l'incanto di ogni apparenza. Si tratta di una verità che non è mai intenzionale. Benché l'artista costruisca un progetto, egli non produce verità o bellezza con l'intenzione di generare o possedere bellezza e verità. La veri- 
tà, ampliando il concetto benjaminiano, è apertura alla relazione e morte dell'intenzione.

«La struttura della verità esige pertanto un essere che, per sua estraneità all'intenzione, somigli a quello puro e semplice delle cose, ma che lo superi per consistenza. La verità non consiste in un intendere che troverebbe nell'empiria la sua determinazione, ma è la potenza che plasma l'essenza di quell'empiria» (Benjamin [1926]: 11) ${ }^{5}$. Come ricorda Raimon Panikkar, stiamo parlando di una verità che non si potrà mai possedere oggettivandola, ma abitare accettando il cambiamento con la massima pienezza nel rapporto con il tutto. La grande difficoltà è cogliere nel cambiamento una verità mutevole, la sua forma che genera forme. L'enigma rimane e noi scorriamo con lui nella vita.

La verità connota sempre una relazione. Quando si distrugge la relazione, si uccide la verità. Se la ricerca della verità non si accompagna a una messa in gioco di quello che io stesso sono, di me che la cerco, allora non è una vera ricerca della verità; è la ricerca di una concettualizzazione o di una dottrina [...]. Ma non è questa verità che ci fa vivere. Quando la verità cessa di essere personale, viva, soggettiva altrettanto che oggettiva, cessa di essere verita. Nessuno ha il monopolio della verità, poiché la verità è pluralista, e non plurale. La verità, come oggetto, non esiste; poiché la verità di una cosa, quando mi sforzo di dirla, è inseparabile dagli interlocutori (Panikkar [1998]: 21).

Vi è un'eloquente metafora che dalle culture persiane, indiane, cristiane ed ebraiche, giunge sino alle geometrie filosofiche spinoziane. È l'immagine della goccia d'acqua come luogo di verità e autenticità, in stretto rapporto con l'eternità e la transitorietà dell'esistenza ${ }^{6}$. Se consideriamo un

\footnotetext{
${ }^{5}$ L'indicazione bibliografica e notazionale rimanda all'edizione qui adottata de Il dramma barocco tedesco (Ursprung des deutschen Trauerspiels, 1926). Tuttavia si precisa che è stato operato un confronto sinottico con la più recente edizione italiana del testo benjaminiano a cura di A. Barale con prefazione di F. Desideri, Carocci, Roma 2018. Da ora in poi, ogni nota relativa al Dramma benjaminiano farà comunque riferimento alledizione Einaudi.

${ }^{6}$ Il riferimento va alle ricerche transculturali e interreligiose di Raimon Panikkar. In un dialogo con Gwendoline
}

mare infinito come il luogo inoggettivabile dell'arte, l'essere umano può essere considerato una goccia in questo infinito. Se rimaniamo in ascolto profondo della nostra natura, noi siamo acqua. Le gocce che si perdono nel mare non fanno scomparire l'acqua di cui sono fatte. Quest'acqua che non posso più differenziare dal tutto ma vivere interiormente non cessa di essere acqua, la mia acqua, l'acqua che io sono. In tale infinito non vi è quindi nessun pericolo di dissoluzione. Sono una goccia d'acqua e nello stesso tempo l'acqua della goccia. L'arte, se così possiamo dire, fa vivere la vita eterna qui e ora in questo infinito mare. E così, nell'accadere di ogni transito, saremo contenuti nella vita eterna di questo infinito. Aristotele ricorda che l'essere umano è un animale in cui transita il logós, è «tõn zõon lógon échon» (Aristotele [2017]: 77), un essere vivente attraversato dalla verità che mai si potrà cogliere in modo definitivo. La grandezza dell'uomo, diceva Nietzsche è di essere infatti «un ponte e non uno scopo: nell'uomo si può amare che egli sia una transizione e un tramonto» (Nietzsche [1885]: 8).

Nel momento in cui la mia transizione si fonde col mio tramontare nasce l'opera d'arte che infiamma questa relazione e nel mio vivere incarno la verità e l'autenticità di questo transito, di questa combustione drammatica che può divenire poetica. Le opere d'arte sono la continua incarnazione di tale verità. L'oggettività che si disegna all'interno della pratica artistica è sempre transitoria ed essendo sempre transito è anche verità del transitare. L'arte è dunque il luogo della verità che transita, è un particolare evento inclusivo, solidale e paticamente vicino alle pratiche che si legano alla trasformazione degli eventi del mondo. Essa stessa è verità contenuta nel bello, nel dolore, nell'autenticità del gesto poetico e in tutte quelle forme che raggiungono il massimo della loro bruciante luminosità?.

Jarczyk, il teologo racconta, in sintesi, la storia culturale della metafora della goccia d'acqua (Panikkar [1998]: 74-75).

${ }^{7}$ Volendo approfondire il complesso tema della verità in Benjamin rimando al saggio di F. Desideri Apocalissi profana: figure della verità in Walter Benjamin in W. Benja- 
Nella verità il momento rappresentativo è il rifugio della bellezza in generale. E infatti il bello rimane provvisto di apparenza, tangibile, finché si riconosce senz'altro come tale. Il suo apparire, che seduce finché non si propone altro che di apparire, si fa perseguitare dall'intelletto, e dà a riconoscere la sua innocenza soltanto laddove si rifugia presso l'altare della verità. Eros lo segue in questa fuga, non persecutore bensi amante, $e$ in modo tale che la bellezza, per salvare il proprio apparire, li fugge entrambi: l'intelletto che la perseguita per timore, lamante per ansietà. É solo quest'ultimo può testimoniare che la verità non è un disvelamento che distrugga il mistero, bensi una rivelazione che gli rende giustizia (Benjamin [1926]: 7).

La verità che fiorisce nel luogo dell'arte non esiste come idea assoluta e oggettivabile poiché quando cerco di dirla è già nel luogo abitato dalla relazione in cui questa verità si muove. Essa è dunque l'incarnazione di una forma partecipativa e potenziale. Grazie a questa forma sensibile riconosciamo le nostre varietà più autentiche e vediamo fuggire questa immagine in un luogo che diffonde dentro sé la propria ombra. Uno specchio nero che illumina se stesso e accoglie le forme nel loro eterno farsi e disfarsi. Una superficie riflettente che ha il coraggio di accettare l'esplosione interna che la attende.

\section{UNA DOLCE RESISTENZA. FORMA E VERITÀ.}

Si fa arte per liberare la vita dalle sue prigioni. Infinite le gabbie in mutevoli verità. Attraverso il linguaggio dell'arte si scava un solco che è differenza di potenziale tra un tutto possibile e i suoi accadimenti. Un bagliore che illumina ciò che stava nell'oscurità, che leviga, che arricchisce le condizioni di possibilità di una vita esausta o rigogliosa. E tuttavia un artista non si può accontentare di una vita sfibrata né di una vita personale. Come ricorda Deleuze nell'atto di fare arte c'è il tentativo di fare della vita qualcosa di più di

min, Angelus Novus. Saggi e frammenti (1962) a cura di R. Solmi, Einaudi, Torino 2014. un fatto personale, di arricchire la vita attraverso una vita.

L'artista o il filosofo hanno spesso una salute fragile, un organismo debole, un equilibrio mal fermo: Spinoza, Nietzsche, Lawrence. Ma non è la morte che li spezza, è bensi l'eccesso di vita che hanno vissuto, provato, pensato. Una vita troppo grande per loro, ma è grazie a loro che "il segno è vicino" (...). Creare non è comunicare, ma resistere (Deleuze [1990]: 163).

Resistere al paradigma informativo dell'attualità, resistere alla mera descrizione della vita, resistere alla morte, al compiacimento dell'arte, alle seduzioni mortifere della violenza. L'artista cerca la verità la cui «fiamma continua ad ardere sui ceppi pesanti del passato e sulla cenere lieve del vissuto» (Benjamin [1962]: 164). In questa fiamma vi è il segreto che avvampa perché l'aria e il legno che ne fanno parte svaniscono per amor di verità. Mentre questa fiamma vacilla, l'artista resiste dolcemente all'accadere del tempo e scompare nella sua opera.

L'artista è dunque trafitto dall'evento e dalla sua potenzialità, dalla verità che transita. In questo passaggio cerca di cogliere l'eterno, poiché l'eterno è nel tempo, è potenza di tempo. È un processo di sintesi che muta e si moltiplica attraverso un'immagine cristallina. Ma l'artista nasconde il significato mostrando il significante di ciò che possiamo chiamare "verità", la sua eterna struttura che continua a ripresentarsi sotto nuova luce. Ogni verità in transito sta in ciò che viene ascoltato non in ciò che viene rivelato, sta nell'abbandonarsi totalmente alla vita, alla poesia che ci parla e che appende ogni preghiera al muro del tempo.

\footnotetext{
Quando vuoi pregare, quando vuoi sapere quel che sa la poesia sporgiti, e senza esitazione cerca il gesto più piccolo che hai, piegalo all'infinito, piegalo fino a terra, al suo batticuore.
} 
Quando hai fame di luce

e l'amore è cinghia serrata

e il cuore stracolmo

di voli che allacciano troppo

al leggero del cielo,

istruisciti alla pura verità,

quella che non vuoi

e nemmeno immagini,

quella della "polvere sul pavimento

e pane sulla tavola",

ginocchia sbucciate

e pane che parla,

dice la fame giusta (Candiani [2014]: 17).

La vita non muore mai, sono piuttosto gli organismi a morire. La vita è un fluire continuo che crea e ricrea se stessa. L'arte illumina sì la vita di un essere umano, ma la trascende perché viene attraversata da infinite vite. Vite che si succedono e in cui ogni accidente singolare mostra la continuità di un'essenza e di un pulsare che sentiamo sconfinati. Si tratta di stare tra la vita e la morte, in una terra di mezzo in cui le nostre esperienze individuali si trasfigurano e la natura umana appare tramontare meravigliosamente. L'artista non ha un volto, poiché la sua opera è nell'immagine di un eterno divenire.

Una vita è ovunque in tutti i momenti attraversati da questo o quel soggetto vivente e misurati da tali oggetti vissuti: la vita immanente porta in sé gli eventi o le singolarità, e questi non fanno che attualizzarsi nei soggetti e negli oggetti. Questa vita indefinita non ha momenti, per quanto vicini siano gli uni gli altri, ma soltanto fra-tempi, fra-momenti. Non sopraggiunge né succede, ma, presenta l'immensità del tempo vuoto dove si vede levento ancora a venire e già arrivato, nell'assoluto di una coscienza immediata (Deleuze [1995]: 11).

In una tale immediatezza si configura una verità che sta nella resistenza potenziale di ogni atto creativo, di ogni singolarità e nel medesimo tempo nella dimenticanza di essere soggetti creativi. L'artisticità diviene nel transito, trova "verità" in questo passare, in questo lasciare andare alla vita. È l'umano che si rigenera inesauribilmente nella forma di ogni forma. Per questo moti- vo «l'artista e l'uomo morale, nella creazione e nell'agire, possono l'uno vedere un nuovo mondo artistico e l'altro procedere verso un nuovo mondo morale soltanto agendo in modo autentico (shinshi)» (Nishida [1965]: 453-454).

Tale autenticità ci porta a guardare un altro volto della verità, in un legame consustanziale tra etica ed estetica. Perdere o negare questo legame significa negare le radici dell'arte, smarrire la relazione con le forme di vita, dimenticare la contemplazione proprio perché trasfigurata nella sua estrema semplicità originaria. Le opere d'arte, in fondo, negano l'arte per riaffermare la vita, nei gesti quotidiani apparentemente banali che possono divenire per noi plurali e politici. L'arte è l'esile filo d'oro che esplode nella vita, un dolce veleggiare ai limiti dell'umano per provare a essere il più umani possibile. Non resta che continuare la ricerca dei fosfeni ${ }^{8}$, accecata memoria di una luce stellare, ai confini di ogni cecità.

\section{REFERENCES}

Aristotele, Politica, a cura di C. A. Viano, BUR, Milano 2017.

Benjamin, W., 1962: Angelus Novus, tr. it. a cura di R. Solmi con un saggio di F. Desideri Angelus Novus. Saggi e frammenti, Einaudi, Torino 2014.

Benjamin W., 1926: Ursprung des deutschen Trauerspiels, tr. it. con introduzione di G. Schiavoni Il dramma barocco tedesco, Einaudi, Torino 1999.

Benjamin W., 1926: Ursprung des deutschen Trauerspiels, tr. it. a cura di A. Barale con prefazione di F. Desideri, Carocci, Roma 2018.

Candiani, C. L., 2014: La bambina pugile ovvero la precisione dellamore, Einaudi, Torino.

Celan P., 1983: Gesammelte Werke, band III tr. it. a cura di G. Bevilacqua La verità della poesia. Il "meridiano" e altre prose, Einaudi, Torino 2008.

\footnotetext{
${ }^{8}$ Fantasmatiche apparizioni luminose che si manifestano alla chiusura o alla compressione delle palpebre. Locchio guarda se stesso nel buio e il lume razionale del logós si fa più vicino ai barlumi fosfenici del poetico.
} 
Deleuze, G., 1995: L'immanance: une vie..., "Philosophie", 47, 1995, pp. 3-7, tr. it. F. Polidori Immanenza. Una vita..., Mimesis, Milano 2011.

Deleuze, G., 1990: Pourparlers, tr. it. di S. Verdicchio Pourparler 1972-1990, Quodlibet, Macerata, 1999.

Deleuze, G., Guattari, F., 1991: Qu'est-ce que la philosophie?, tr. it. di A. De Lorenzis, a cura di C. Arcuri Che cosè la filosofia, Einaudi, Torino 1996.

Derrida, J., 1967: De la grammatologie, tr. it. a cura di G. Contri, G. Dalmasso, A.C. Loaldi Della grammatologia, Jaca Book, Milano 1969.

Derrida, J., 1972: La dissémination, tr. it e cura di S. Petrosino La disseminazione, Jaca Book, Milano 2018.

Desideri, F., 2014: Apocalissi profana: figure della verità in Walter Benjamin in Benjamin, W., 1962 in Angelus Novus. Saggi e frammenti, tr. it. R. Solmi, Einaudi, Torino 2014.

Ghilardi, M., 2018: La filosofia giapponese, Morcelliana, Brescia.

Ghilardi, M., 2009: L'unità di buono, vero e bello nell'estetica di Nishida Kitarō, in Premio nuova estetica, a cura di L. Russo, Aesthetica Preprint, Palermo.

Morin, E., 2016: Sur l'esthétique, tr. it. di F. Bellusci Sullestetica, Raffaello Cortina Editore, Milano 2019.

Nietzsche, F., 1885: Also sprach Zarathustra. Ein Buch für Alle und Keinen, tr. it. con nota intro. G. Colli Cosi parlò Zarathustra. Un libro per tutti e per nessuno, Adelphi, Milano 2017.

Nietzsche, F., 1873: Über Wahrheit und Lüge im auBermoralischen Sinne, tr. it. G. Colli con un saggio di B. Zavatta Su verità e menzogna in senso extramorale, Adelphi, Milano 2015.

Nishida, K., 1926: Basho 場所, in “Tetsugaku kenkyū" [Ricerche filosofiche], n. 123, giugno tr.it. a cura di E. Fongaro e M. Ghilardi Luogo, Mimesis, Milano-Udine 2012.

Nishida K., 1965: Opere complete di Nishida Kitarō zenshu 西田幾多郎全集, Iwanami shoten, Tōkyō 2008.

Panikkar, R.,1998: Enre Dieu et le cosmos. Une vision non dualiste de la réalité, tr. it. a cura di
M. Sampaolo Tra Dio e il cosmo. Dialogo con Gwendoline Jarczyk, Laterza, Roma-Bari 2019.

Platone, 2000: Critone, a cura di G. Reale, Bompiani, Milano.

Platone, 2015: Fedro, a cura di S. Mati, Feltrinelli, Milano.

Raveri, M., 2014: Il pensiero giapponese classico, Einaudi, Torino.

Robin, L., 1968: Platon, tr. it. e cura di F. Calabi Platone, Cisalpino, Milano 1988.

Silesius, A., 1905: Cherubinischer Wandersmann, tr. it. e a cura di G. Fozzer e M. Vannini Il pellegrino cherubico, San Paolo, Milano, 1989.

Wittgenstein, L., 1961: Tractatus logico-philosophicus, tr. it. e a cura di A.G. Conte, Tractatus logico-philosophicus e Quaderni 1914-1916, Einaudi, Torino 1998.

Zanzotto, A., 2011: Tutte le poesie, a cura di S. Dal Bianco, Mondadori, Milano. 